\title{
Autoimmune limbic encephalitis: a reversible form of rapidly progressive amnesia and seizures
}

\author{
${ }^{1} \mathrm{~A}$ Ahmad, ${ }^{2} \mathrm{~S}$ Ramakrishna, ${ }^{3} \mathrm{~J}$ Meara, ${ }^{4} \mathrm{M}$ Doran \\ ${ }^{1}$ Specialist Registrar (ST3), Geriatric Medicine, North Manchester General Hospital, Crumpsall; ${ }^{2}$ Consultant Physician, Caerphilly District $\mathcal{E}$ \\ Miners' Hospital, Caerphilly; ${ }^{3}$ Senior Lecturer, Academic Department of Geriatric Medicine, Cardiff University, Glan Clwyd Hospital, Rhyl, \\ North Wales; ${ }^{4}$ Consultant Neurologist, The Walton Centre for Neurology \& Neurosurgery, Fazakerley, Liverpool, UK
}

\begin{abstract}
Many forms of rapidly progressive cognitive impairment are irreversible and rarely treatable. We present a case of autoimmune limbic encephalitis associated with voltage-gated potassium channel autoantibodies that led to rapid cognitive deterioration and bizarre behaviour, which subsequently improved following immunosuppressant therapy. The diagnosis of autoimmune limbic encephalitis needs to be considered in patients presenting with apparent viral encephalitis, in more unusual clinical situations in subjects with acute psychiatric illness and in cases of refractory epilepsy, particularly if there is evidence of amnesia. The diagnosis depends on the detection of specific antibodies directed against central nervous system targets. There can be a good response to immunosuppressant therapy.
\end{abstract}

Correspondence to A Ahmad, 4 Rhodfa Conway, Dyserth, Rhyl, North Wales LLI 8 6LS, UK

tel. +44 (0) I 745570355

e-mail dr.amirahmed@gmail.com

KEYWORDS Autoimmune encephalitis, cognitive impairment, limbic system, voltage-gated potassium channel autoantibodies

DECLARATION OF INTERESTS No conflict of interests declared.

\section{CASE REPORT}

A 63-year-old retired schoolteacher was admitted to the acute medical unit from an inpatient psychiatric unit following a generalised tonic-clonic seizure. He had been found wandering in the street in an unkempt state and, as he was unable to give any personal details to the police, was sent to the psychiatric unit and detained under the Mental Health Act. He was diagnosed in the psychiatric unit with dementia, but four days after his admission there he had a seizure that necessitated his admission to the medical unit.

Prior to his admission the patient lived independently on his own and appeared to have no significant medical or psychiatric history or history of excessive alcohol intake. On admission he appeared to be disorientated in time and place and had a marked impairment of short-term memory. He scored $20 / 30$ on the mini mental state examination, although mental arithmetic and higher executive functions were preserved. Physical examination was normal. Investigations, including serum electrolytes, glucose, thyroid function tests and serum vitamin $\mathrm{BI} 2$, were normal. A computed tomography (CT) brain scan was also reported as normal.

More history was obtained from the patient's sister, who said that four weeks previously he had driven a long distance to care for her when she was recovering from an eye operation and he had been in charge of administering her eye drops. Further enquiry revealed that his neighbours had noticed over the three-week

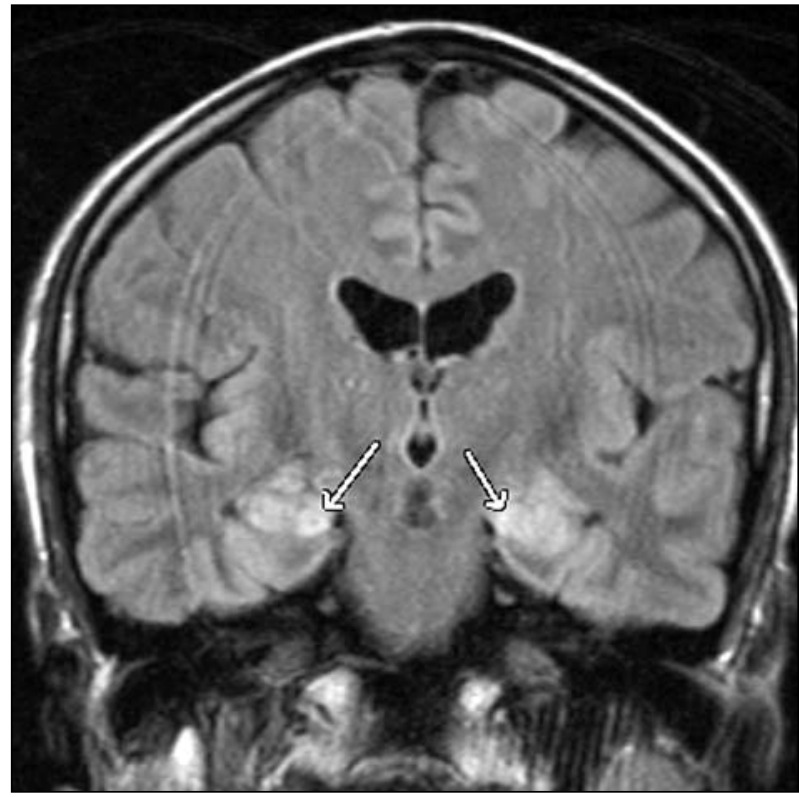

FIGURE I Magnetic resonance image of the patient's brain, showing bilateral high-intensity signals in the hippocampi consistent with limbic encephalitis (see arrows).

period prior to his admission that his behaviour had become odd and out of character. He tended to lose track of his conversation and appeared to wander aimlessly around the neighbourhood.

A lumbar puncture showed normal cerebrospinal fluid (CSF) biochemistry and sterile culture. The CSF was sent for protein 14-3-3 for Creutzfeldt-Jakob disease and polymerase chain reaction for herpes simplex and 
other viruses. All of these were subsequently negative. The patient's electroencephalogram was normal. A magnetic resonance imaging (MRI) brain scan revealed high-intensity signal in the limbic area bilaterally on T2-weighted images (Figure I).

On the basis of the clinical history, examination and neuroimaging, limbic encephalitis was considered a likely diagnosis. This was confirmed by the presence of high titres of serum anti-voltage-gated potassium channel autoantibodies (anti-VGKC) $(1,475 \mathrm{pM}$, normal $<100)$ in a radioimmunoprecipitation test done at Professor Angela Vincent's laboratory at the Weatherall Institute of Molecular Medicine, Oxford. The patient also underwent a CT scan of his thorax, abdomen and pelvis with contrast to look for tumours causing paraneoplastic limbic encephalitis, but the scan was normal.

He was started on immunotherapy comprising intravenous immunoglobulin $(0.4 \mathrm{mg} / \mathrm{kg}$ for five days), oral prednisolone (60 mg, tapered off over eight weeks), and plasmapheresis. The patient responded to this treatment and within a week was better oriented and showed significant improvement in his cognitive state. The titres of the autoantibody fell progressively to undetectable levels over a period of three weeks following the initiation of treatment.

A repeat MRI brain scan showed definitive improvement radiologically of his hippocampus. The patient remained free of symptoms at six-month follow-up, but after one year had another relapse in the form of abnormal behaviour. An anti-VGKC antibody test came back raised on this occasion as well, and he received treatment in the form of a five-day course of methylprednisolone. He improved following treatment, but in view of the relapse he was put on maintenance prednisolone, $40 \mathrm{mg}$ once a day, with a view to reduce the dose in future. Since then the patient has been under regular follow-up by a neurologist, with no further relapses.

\section{DISCUSSION}

Limbic encephalitis can be caused by infections or result from autoimmune disease. Several viruses, most notably herpes simplex, can cause inflammation of the limbic area of the brain. Autoimmune limbic encephalitis can be part of a paraneoplastic process occurring in a small proportion of patients with lung, thymus, breast and testicular malignancies. Diagnosis can be made by detecting paraneoplastic autoantibodies such as anti-Hu (lung tumours) and anti-Ta (testicular tumours). Paraneoplastic encephalitis does not usually respond to immunosuppression, unlike anti-VGKC encephalitis. Limbic encephalitis can also be associated with specific anti-VGKC autoantibodies, first described by Buckley et al. in 2001.'
Unlike paraneoplastic encephalitis, anti-VGKC-associated encephalitis is potentially treatable and reversible. The reported median age of onset is 64 years (age range 44-79 years), with men more commonly affected than women in the two initial case series. ${ }^{2.3}$ All reported cases of anti-VGKC encephalitis had acute or subacute cognitive and behavioural changes, most commonly impaired short-term memory, inattention and confusion. Visual hallucinations, myoclonic jerks and dystonic facial movements have also been reported. ${ }^{24}$ Seizures have frequently been reported and cases of refractory epilepsy have also been subsequently diagnosed with anti-VGKC encephalitis.

Patients presenting with behavioural symptoms have been erroneously admitted to psychiatric units, until a precipitating seizure alerts the possibility of an underlying medical rather than psychiatric cause. ${ }^{5,6}$ The diagnosis of anti-VGKC encephalitis could easily be overlooked in patients treated empirically for presumed herpes simplex encephalitis in culture-negative cases or when specimens have not been sent for viral testing.

Hyponatraemia, hypothesised to be secondary to inappropriate antidiuretic hormone release, is commonly reported and may contribute to cognitive impairment and seizures, although patients can have these even with normal sodium levels. ${ }^{2.7}$ The change in serum anti-VGKC antibody titres corresponds to the degree of neurological dysfunction, with clinical improvement being reflected in falling autoantibody titres. ${ }^{8}$ The CSF proteins may be mildly elevated, and although autoantibodies to voltagegated potassium channels may be detected in the CSF, the serum levels of the autoantibody (anti-VGKC) are usually diagnostic. The white cell count in CSF may be slightly increased or even normal, and normal CSF findings do not preclude VGKC limbic encephalitis."

The CT brain scan is usually normal, although the MRI shows mesial temporal lobe abnormalities, usually bilaterally with increased signal on $\mathrm{T} 2$ and fluid-attenuated inversion recovery images. Other areas of abnormality on the MRI scan, such as the inferior frontal lobe, anterior caudate and corpus callosum, have also been described, but are uncommon. ${ }^{4}$

Immunosuppressive therapy with high-dose intravenous methylprednisolone, intravenous immunoglobulin or plasmapheresis, often in combination, can prevent further neurological damage and lead to significant clinical improvement in patients treated within two months of disease onset. ${ }^{2,3}$ The search for a tumour by imaging is essential to rule out paraneoplastic limbic encephalitis, even if VGKC antibodies are demonstrated. ${ }^{10}$ In the Jarius et al. study, six out of 27 (22\%) VGKC antibodypositive patients had tumours, compared with 32 out of 4 I $(78 \%)$ with paraneoplastic antibodies. ${ }^{10}$ 
If the clinical picture is not clear, other onconeural antibodies should be tested for paraneoplastic limbic encephalitis. One particular condition that can mimic the presentation of anti-VGKC antibody encephalitis is the anti-N-methyl-D-aspartic acid receptor (anti-NMDAR) encephalitis. This condition is more commonly found in younger patients, particularly women with ovarian teratoma. Behavioural problems and personality changes are prominent." Serum and CSF NMDAR antibodies are present, the CSF shows lymphocytic pleocytosis and the MRI can be normal, show transient cerebral cortical or cerebellar abnormalities, or show typical medial temporal lobe hyperintensities. ${ }^{12,13}$ The condition is treated with tumour removal, immunosuppression and intensive care. An understanding of the pathophysiology of these conditions is still unfolding gradually. There is recent, unpublished evidence that VGKC-associated limbic encephalitis is due to antibodies directed against proteins that are associated with the VGKC and not directly against the VGKC.

\section{REFERENCES}

I Buckley C, Oger J, Clover L et al. Potassium channel antibodies in two patients with reversible limbic encephalitis. Ann Neurol 200I 50:73-8. doi: 10.1002/ana. 1097

2 Vincent A, Buckley C, Schott JM et al. Potassium channel antibodyassociated encephalopathy: a potentially immunotherapyresponsive form of limbic encephalitis. Brain 2004; 127:701-12. doi:I0.1093/brain/awh077

3 Thieben MJ, Lennon VA, Boeve BF et al. Potentially reversible autoimmune limbic encephalitis with neuronal potassium channel antibody. Neurology 2004; 62: I I77-82.

4 Tan KM, Lennon VA, Klein CJ et al. Clinical spectrum of voltagegated potassium channel autoimmunity. Neurology 2008; 7:188390. doi: I0.12I2/0I.wnl.00003 I2275.04260.a0

5 Parthasarathi UD, Harrower T, Tempest $M$ et al. Psychiatric presentation of voltage-gated potassium channel antibodyassociated encephalopathy. $\mathrm{Br} J$ Psychiatr 2006; 189:182-3. doi:I0.I 192/bjp.bp. I05.0I2864

6 McKen A, Marnane M, O'Connell M et al. Potassium channel antibody-associated encephalopathy presenting with a frontotemporal dementia-like syndrome. Arch Neurol 2007; 64:1528-30. doi:10.1001/archneur.64.10.1528

7 Schott JM. Limbic encephalitis: a clinician's guide. Pract Neurol 2006; 6:143-53. doi:10.1136/jnnp.2006.091827

\section{CONCLUSIONS}

This case highlights the need to increase awareness about autoimmune limbic encephalitis, particularly when it presents as an apparent psychiatric emergency or with refractory epilepsy. Acute/subacute cognitive impairment, especially with accompanying memory loss with seizures and no other identifiable diagnosis (for example, a normal CT head scan and normal lumbar puncture), should suggest the possibility of autoimmune limbic encephalitis. The condition can usually be diagnosed with simple tests such as the anti-VGKC antibody and an MRI scan. Other forms of limbic encephalitis, such as viral and paraneoplastic, should be ruled out during the investigations. The prognosis is good with prompt treatment in the form of immunosuppression.

8 Dunstan EJ,Winer JB.Autoimmune limbic encephalitis causing fits, rapidly progressive confusion and hyponatraemia. Age Ageing 2006; 35:536-7. doi: 10.1093/ageing/afl045

9 Jarius S, Hoffmann L, Clover L et al. CSF findings in patients with voltage gated potassium channel antibody associated limbic encephalitis. J Neurol Sci 2008; 268:74-7. doi:10.1016/j. jns.2007.II.004

10 Jarius S, Hoffman LA, Stich O et al. Relative frequency of VGKC and 'classical' paraneoplastic antibodies in patients with limbic encephalitis.J Neurol 2008; 225: I I00-0I. doi:10.1007/s004 I5-0080845-3

II Wilder-Smith EP, $\mathrm{Ng}$ ES. The writing on the wall. Lancet 2008; 26:344. doi:10.1016/S0I40-6736(08)6II20-4

12 Dalmau J, Gleichman AJ, Hughes E et al. Anti-NMDA-receptor encephalitis: case series and analysis of the effects of antibodies. Lancet Neurol 2008; 7:109I-8. doi:I0.1016/SI474-4422(08)70224-2

13 Sansing LH, Tüzün E, Ko MW et al. A patient with encephalitis associated with NMDA receptor antibodies. Nat Clin Pract Neurol 2007; 3:291-6. doi:10.1038/ncpneuro0493 